\title{
Technology Research for Rotor Wedge Installation of Hydrogenerator
}

\author{
Zhou Ruoyu \\ Manufacturing and Installation Branch, Sinohydro Engineering Bureau 3 Co., Ltd., Xi' an, China \\ Email address: \\ Dzry1983110@163.com \\ To cite this article: \\ Zhou Ruoyu. Technology Research for Rotor Wedge Installation of Hydrogenerator. American Journal of Water Science and Engineering. \\ Vol. 7, No. 3, 2021, pp. 97-102. doi: 10.11648/j.ajwse.20210703.11
}

Received: May 30, 2021; Accepted: June 17, 2021; Published: July 8, 2021

\begin{abstract}
When the rotor of the hydroelectric generator is running, the centrifugal force of the rotor rim is very high. Centrifugal force will produce obvious radial deformation on the rotor rim lamination, which will cause a radial separation gap between the rim and the rotor spider. The larger the diameter of the rotor, the larger the separation gap, therefore, when the rotor is assembled, each component is required to have sufficient strength, good balance and integrity. In order to ensure the stability and balance of the rim during the installation, it is necessary to focus on the technical measures to control installation of the wedges, to complete the thermal keying operations in strict accordance with the requirements. The purpose of the rotor thermal keying is to maintain the safety and stability during operation, to facilitate rim and rotor hub to be closely combined, and to achieve a reliable cooperation between them. A certain amount of mechanical compression is applied simultaneously between rim and rotor spider, which can generally be achieved by thermal keying or conventional rim hot keying on the basis of cold keying, the rim is heated to generate gap between rotor rim and hub, the keys are driven to the gap in specified depth. After the rotor rim is cooled down, the effective coordination between rotor rim and rotor hub can be ensured, the centripetal force generated during the operation of the unit can be offset to maintain the unit's reliable operation. The installation of hydro-generator rotor's wedge is an important process, it's a matter of the roundness and concentricity, higher requirements is needed for large capacity generators. Based on the structural characteristics of the rotor installed at the Ludila Power Station on the Jinsha River, this article introduces the installation and welding process of the wedges for the rotor at the Ludila Power Station, as well as the difference between thermal keying technology and conventional rim hot keying. Quality control, installing procedure and methods for installation of rotor wedge are described. This article could provide guidance and reference for the implementation of similar projects, research on new advanced technology for the industry.
\end{abstract}

Keywords: Hydrogenerator, Rotor Rim, Wedge, Installation Procedure, Ludila Hydropower Station, Thermal Keying

\section{Introduction}

When the rotor of the hydroelectric generator is running, the centrifugal force of the rotor rim is very high. Especially for large-scale units, the huge centrifugal force will cause obvious radial deformation on the rim lamination, which will cause a radial separation gap between the rim and the rotor support [1]. The larger the rotor diameter, the larger the separation gap. Therefore, the rotor components are required to have sufficient strength, good balance and integrity during installation. With more large-capacity units been applied in projects, to ensure that the rim and the rotor hub will not produce a separation gap when the unit is operating at the separation speed of the unit, measures against gap have been applied during the assembly of the rotor, especially the installation of Rotor wedge and the thermal keying for rotor $\operatorname{rim}[2-6]$.

Thermal keying is an important step to the rotor installation process. In order to ensure the safety and stability of the unit during operation, the rim and the rotor hub must be closely combined to achieve an interference fit. A certain amount of mechanical compression is required to apply to the rotor rim and the rotor spider. During the assembly process, a pre-tightening force must be applied to the rim and the rotor spider in advance [7]. Thermal keying and is usually used in the assembly of hydro-generator rotors. Thermal keying is based on cold keying, on heating, rim expands to generate the gap between the rim and the rotor hub. After the rim is 
cooled, the rim and the rotor hub are interference-fitted to produce centripetal force, which can offset the centripetal force during the operation to ensure the long-term safe and stable operation [2, 8-10]. Traditional thermal keying is generally used for small-capacity, low-speed operating units, which could cause uneven force. To eliminate uneven force, most large-scale hydro-generator rotor assembly adopts hot padding method. In view of the rotor structure at Ludila power station, wedge structure and thermal keying are connected and fixed with the rim for installation.

\section{Structure Characteristics of Rotor}

Ludila Hydropower Station is equipped with 6 hydro-generators with a rated speed of $100 \mathrm{r} / \mathrm{min}$ and $\mathrm{a}$ capacity of $360 \mathrm{MW}$. The structure of generator is vertical shaft semi-umbrella. The rotor consists of rotor hub, spider, wedge, rim, and pole. When assembling on site, the rotor hub and spiders are integrated and welded together. The hot cushion sleeve to be installed when rim is on heating, then the rim is hot sleeved on the rotor spider and forms a whole with the rotor spider. The rotor spider is assembled and welded into the hub. The support arm sector are made by welding 20 wedges are on site. Each single wedge weighs $621 \mathrm{~kg}$ and $2487 \mathrm{~mm}$ in height. The outer diameter of the rotor is $\Phi 14063.3 \mathrm{~mm}$, the height is $2574 \mathrm{~mm}$, and the total weight of the rotor is $847150 \mathrm{~kg}$. The rotor structure is shown in Figure 1.

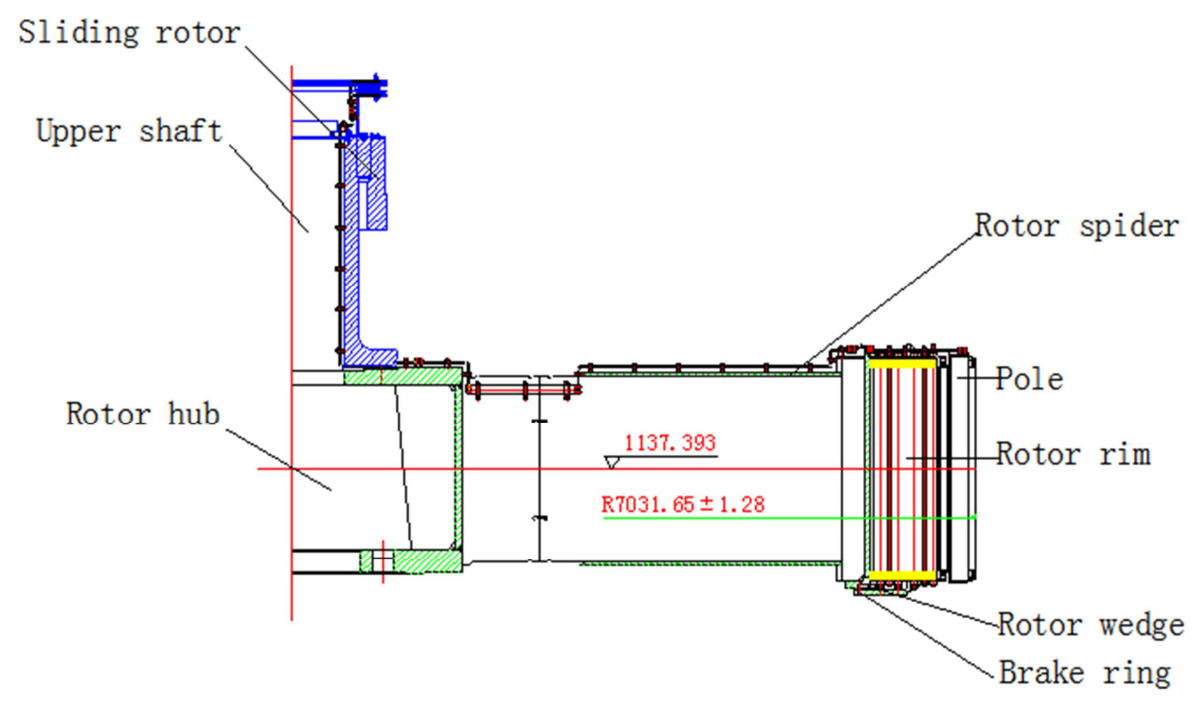

Figure 1. Rotor structure diagram.

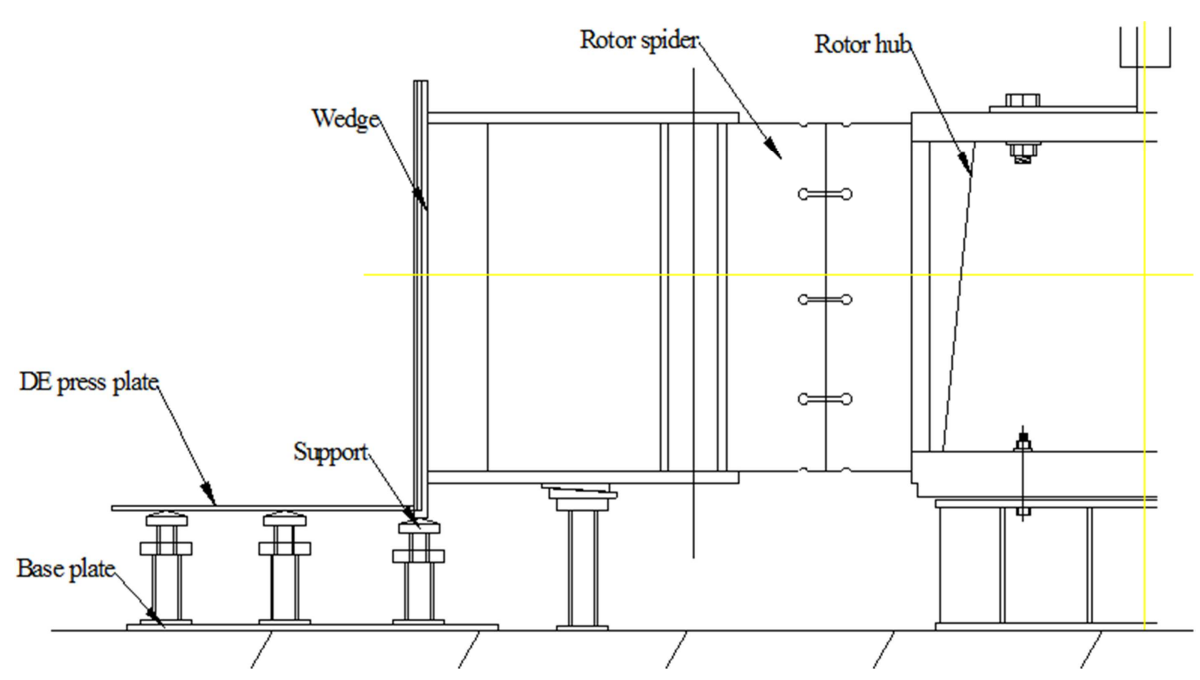

Figure 2. Rotor wedge and lower plate of rim support diagram.

\section{Rotor Wedge Assembly}

\subsection{Installation Process}

(1) Lifting Rotor wedge

Use special tools to hoist the wedge, lower wedge down on its supports, and temporarily spot weld them to the rotor spider with turnbuckle bolts, as shown in Figure 2.

During the alignment and fit-up, the height difference between wedge lifting hook for the rotor and the lower flange of the rotor hub is designed to be $73 \mathrm{~mm}$, the height difference is adjusted to $75 \mathrm{~mm}$ in consideration of the 
self-weight of the rim stack. After the alignment is completed, using the inner diameter micrometer and the measuring frame to measure and adjust the distribution radius of the wedge to meet the design requirements with using inside micrometer. Considering welding shrinkage, the distribution radius of the wedge before welding shall be $3 \mathrm{~mm}$ larger than the design value. The verticality of the wedge in the radial and circumferential directions to be adjusted within $1.0 \mathrm{~mm}$ with using wire-hanging hammer.

(2) Adjustment of the benchmark wedges

1) Take the wedge in the $+Y$ direction as the reference wedge, and measure and adjust its radius to meet the requirements with inside micrometer;

2) Hang the piano wire to measure and adjust its circumferential and radial verticality within $0.15 \mathrm{~mm}$.

(3) Adjustment of wedges

The adjustment of the wedges is determined according to the size of the rim stacking. After the wedges are mounted, pre-stacking of rim to be performed according to the rim stacking diagram and the rim stacking table, the pre-installed rim height is set to be $100 \mathrm{~mm}$. Take the $+\mathrm{Y}$ direction as the starting point, measuring the radius and roundness of the pre-stack rim in clockwise to meet the requirements with inside micrometer. Insert the guide pins coated with molybdenum disulfide into the rim respectively, to ensure the guide pin can be turned free by hand, otherwise, reshaping is required.

Repeating adjustment of rim radius and roundness until meet the requirements, use the same method as above mentioned to check that benchmark-wedge's radius, perpendicularity, and elevation to meet the requirements. After the adjustment of the benchmark wedges is qualified, the following methods are to adjust other wedges:
1) Rotate the supporting screw at the bottom of the wedge to adjust the height difference between it and the lower flange surface of the rotor hub to meet the requirements;

2) Put two dial gauges on the measuring circle frame, starting from the upper and lower points of the reference wedge, adjust the radius and radial verticality of other wedges to meet the requirements;

3) Taking the midpoint of benchmark wedge on top of the rim keys the starting point, measure the chord distance between adjacent wedges to meet the requirements.

After completion of the adjustment for wedges, stacking of the rim could be carried out.

\subsection{Welding Process}

The welding of wedges is an important step to installation of wedges. The quality of welding directly affects the installation of the wedges and the roundness of the rim. Therefore, before the wedges are welded, the lap board (lap board size: $\delta 30 \times 100 \times 200)$ to be arranged at the back seam of the welding seam in the upper, middle, and lower positions as shown in Figure 3 to prevent the excessive shrinkage during welding. In order to monitor the deformation, punch points for measuring the welding deformation (shrinkage) are marked on both sides of each weld joint before welding (as shown in Figure 4). The weld shrinkage, rim radius, roundness, the levelness of rotor hub to be monitored respectively in the following stages, before and after reinforcement of wedge, welding progress on $25 \%, 50 \%$, $75 \%, 100 \%$ and after cooling down.
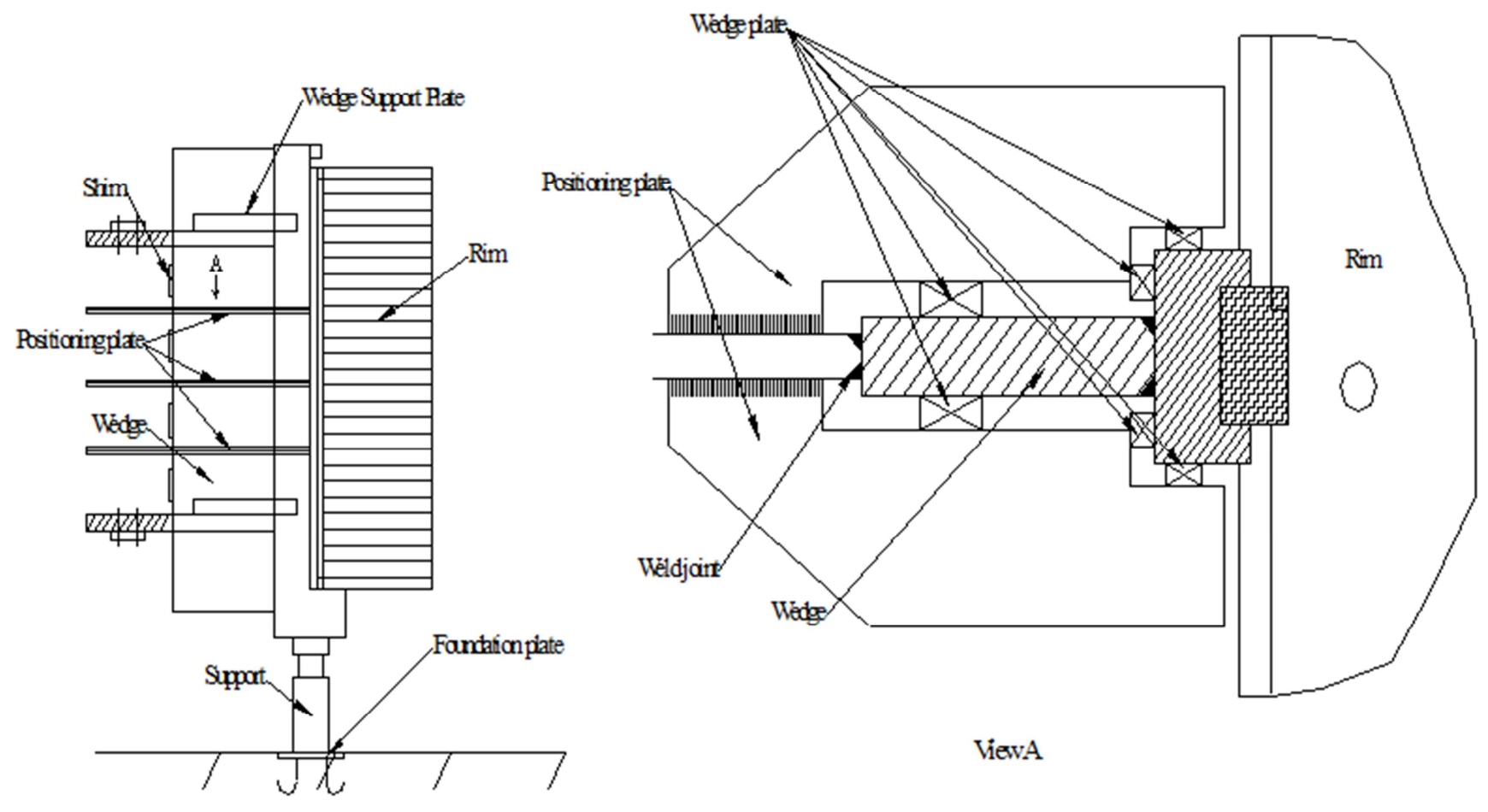

ViewA

Figure 3. Schematic diagram of the layout of the welding positioning plate. 


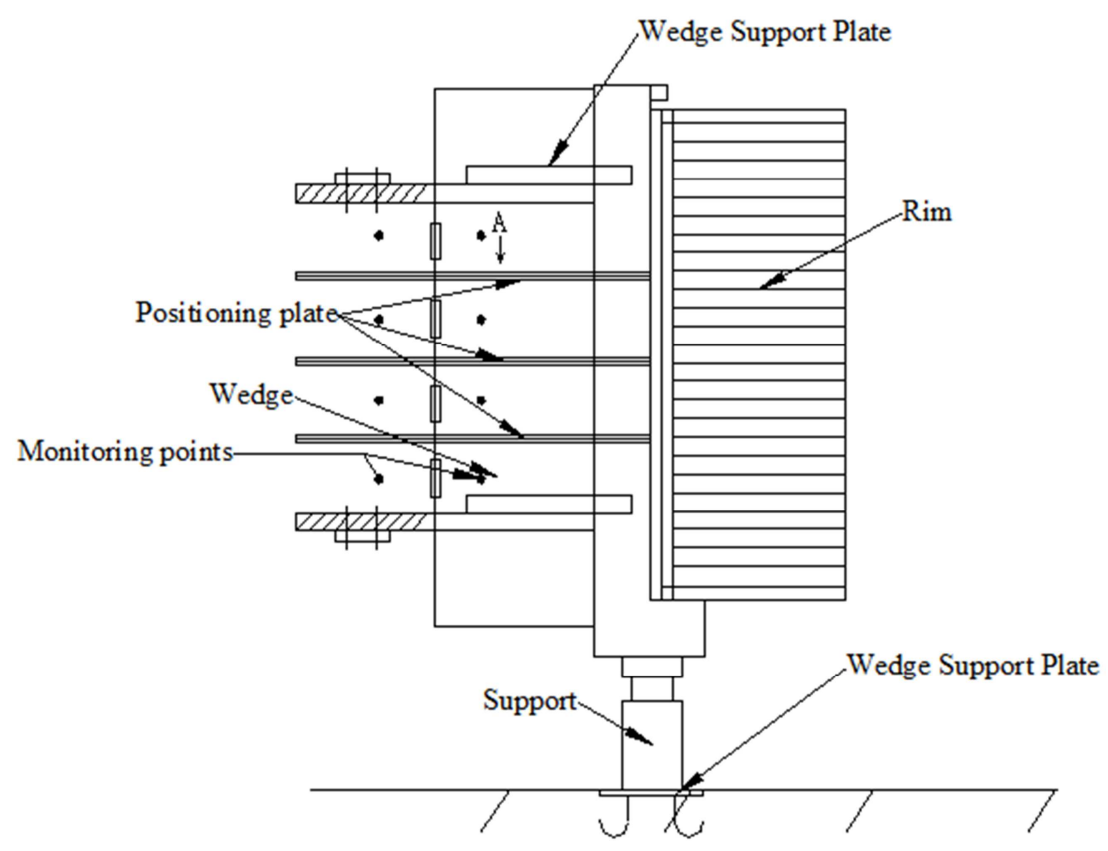

Figure 4. Schematic diagram of the layout of the welding monitoring point of the wedge.

During the welding of wedge, the temperature between layers shall be below $250^{\circ} \mathrm{C}$, whole process of welding shall be no break. The welding of seam requires multi-layer and multi-pass welding, the thickness of deposited metal for each layer shall be less than $5-6 \mathrm{~mm}$ and the joints between each layer must be staggered by $30-50 \mathrm{~mm}$; the welding seam requires short arc operation, narrow and small swing, the surface width of deposited metal for each layer shall be less than $20 \mathrm{~mm}$. Except for the bottoming and covering, the other layers of welding must be hammered and vibrated with a wind handle [11].

Before welding of each wedge, keep monitoring the changes of the wedges during the bottoming and filling process of each welding, welding sequence and volume shall be adjusted immediately when deformation is out of expectation.

After the wedges are welded and the NDT testing are completed, check the levelness of the rotor hub, the round frame, radius and roundness of the rims. Measure the verticality of the rims at the magnetic pole of $1 \#(+Y$ direction) by hanging the piano wire in parallel, and calculate the radial verticality of the rims at other positions based on the radius data; at the same time, measure the gap between the wedge and the rim, detail record shall be maintained as basis for rotor rim thermal keying.

\section{Rotor Rim Thermal Keying}

The process of rotor rim Thermal keying is by heating the rim to generate a gap between wedge and rim, filling the gap with shim, cooling down the heated parts, the rim and the bracket could tightly fitted due to shrinkage during the process of cooling.

\subsection{The Difference Between Thermal Keying and Conventional Keystroke}

The principle of conventional rim thermal keying is similar to method for installation of wedge as mentioned at part 3 . After the rim is stacked, the main and the auxiliary key of the rim are assembled. After the rim is heated, the main and the auxiliary key are tightened to the specified depth. When the rim key is driven into the depth corresponding to the radial deformation increment calculated by the design under the hot state of the rim, the rim usually couldn't generate the expected tightness. Thermal keying is a brand-new method by adding pad between rim and wedge during heating. Compared with conventional thermal keying, the thermal keying method could handle the gap properly between rim and wedge, and easily to be applied on site to meet the surface combination.

\subsection{Method of Rim Heating}

The key to the successful rim thermal keying is by heating rim to generate a sufficient temperature difference between rim and wedges. The heating methods are as follows: (1) Copper loss method. The copper loss method is to connect the pole windings that have been mounted in series, charged with direct current to heat the rim. It is only suitable for calculating the rim with a small temperature difference. (2) Iron loss method. The iron loss method is to apply winding around the rim before the pole is mounted, and charged with alternating current to heat rim. (3) Electric heating method. The electric heating method is to evenly arrange a special electric heater or far-infrared heating at the bottom of the rim and the ventilating trench [12-14].

Appropriate heating method should be selected according to the specific parameters and the actual condition on site. Electric heating method is adopted at Ludila power station.

\subsection{Thermal Keying}

The rotor rim at the Ludila Hydropower Station using 
thermal key (hot jacket) structure, as shown in Figure 5. The theoretical value of thermal pad is $\mathrm{t}=3.4 \mathrm{~mm}$, and the process is as follows:

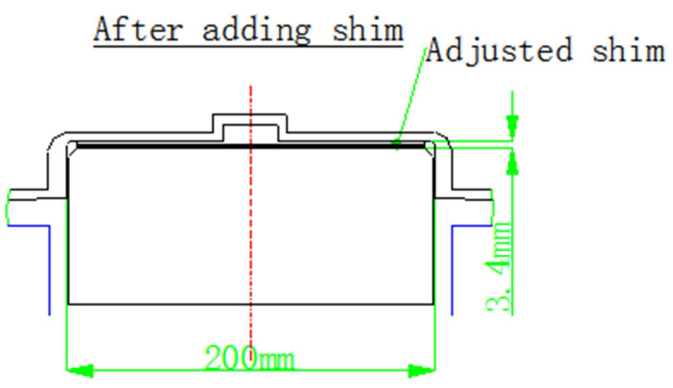

Figure 5. Schematic diagram of rim thermal key structure

(1) After rim lamination and rotor spider are welded, measure the gap " $d$ " between the rim key and the wedge of each rotor spider, and mark them separately;

(2) Calculate the thickness of the adjustment shim $\mathrm{t} 1=\mathrm{t}+\mathrm{d}$, configure the adjustment shim; Calculate the thickness of the shim contracted by the rotor rim, take the value of the gap measured at the top 1, top 2, middle, bottom 1 , and bottom 2 to plus shrinkage value. If the difference between the upper, middle, and lower measured gaps is greater than $0.3 \mathrm{~mm}$, consider preparing shims with different thicknesses for the upper, middle, and bottom.

(3) The rim is heated to radial expansion about $4.1 \mathrm{~mm}$ (the radial amount increases by $0.7 \mathrm{~mm}$ after keying), the reference heating temperature is $\mathrm{T}=$ room temperature $+75^{\circ} \mathrm{C}$, and the heating temperature rise is controlled at $6-10^{\circ} \mathrm{C} / \mathrm{H}$, After the rim expands, immediately insert the adjusting shim, and tighten the rim after cooling.

After the rim thermal key is completed, slowly lower the rim temperature to room temperature, record the corresponding temperature at the cooling time interval, and ensure that the temperature difference within the circumference is not greater than $20^{\circ} \mathrm{C}$, cooling time must meet (not less than) the design requirements. After cooling, the roundness, radius and radial perpendicularity of the rim to be measured to meet the requirements.

After cooling, measure the distance between the upper pressing plate of the rim and the top surface of the upper groove of the wedge. According to the measured data, calculate the thickness of the shim required between the locking plate and the upper pressing plate, and install the wedge locking plate.

\section{Key Points and Difficulties in Installation of Wedge}

(1) Size control

When installing and adjusting the wedge, pay attention to the following points:(1) After the adjustment of the benchmark wedge, the chord distance and radial verticality of the wedges will affect each other when adjusting, The adjustment of the chord distance and radial verticality of the vertical ribs should be carried out simultaneously. (2) The size of the wedges are controlled and adjusted by the size of the rim. Therefore, after the installation and adjustment of the wedges, part of the rim to be pre-installed, the wedges to be checked and adjusted under the condition of ensuring the roundness of the rim. The wedge shall be completely vertical, no radial gap between the rim and the wedges. Every time when the rim is compressed, after inspection of the rim is completed, the wedges should be inspected according to the above requirements.

(2) Welding process control

During the welding of wedge, the deformation of the wedge should be monitored at any time, and the welding sequence should be changed according to the deformation in order to reduce the deformation. The welding should be interrupted when necessary. When the weld joint is slowly cooled down to ambient temperature under the asbestos coverage, welding sequence could be adjusted according to the verticality of the wedges in the axial and circumferential directions.

At the same time, the following points should be noted: (1) In order to reduce the deformation, the welding joint of wedges are welded with $\varnothing 3.2$ welding rod. (2) During welding, the weld bead is distributed from the two sides first and then the middle.

(3) Thermal keying temperature control

Rim to be heated to specified temperature with increment of less than $10^{\circ} \mathrm{C}$, otherwise it should be adjusted. Due to the long heating time and heat conduction, the temperature of the rotor spider and the wedge will rise and expand along with rim heating, expected gap between the rim and the wedge may not be generated, water spraying on the rotor spider to reduce the temperature is a better solution.

After the thermal keying is completed, slowly lower the rim temperature to ambient temperature for at least 48 hours.

The temperature is recorded every 30 minutes, including the upper, middle, and lower parts of the rim and the outside of the rim, as well as the top and bottom surfaces of the rim and the rotor spider, measuring one point per pole in the circumferential direction.

\section{Conclusion}

As more large-scale hydropower stations are applied in China. There are ways of rim fastening and rim keying. Most 
of the traditional power station rotor assembly adopts the rim key method. The rim key can only meet the requirements of small capacity and low speed operation, so large Hydropower stations usually use wedge and thermal keys. The use of wedges and thermal keying can more effectively eliminate the influence of factors such as uneven rim lamination, ensure that the rim reaches the expected tightness, and meet the requirements of safe operation of the unit [13]. Installation of the rotor wedge for the hydro-generator determines the operating efficiency of the commissioning to a large extent. Technical specification on every step to be followed during installation $[14,15]$.

After inspection for installation of the rotor at Ludila power station, all steps meet the specification requirements, the overall quality of the rotor is well controlled. The method for installation of rotor wedge and thermal key at Ludila hydro-power station as mentioned above could be a reference to similar projects during the installation of hydro-generator rotor.

\section{References}

[1] Edited by Harbin Institute of Electrical Engineering. Hydrogenerator design and calculation

[2] Yang Guangyu, Zheng Kai, Tong Fan. (2020). Assembly of generator rotor support and quality control of welding deformation [J]. Yangtze River, v. 51 (S2): 255-259.

[3] ZhangWeiyong, LiChangyun. (2019). Rotor assembly technology of hydro-generator unit of Dahuaqiao Hydropower Station [J]. Water Power, 045 (006): 104-107.

[4] ZouWeihong, Hu Xiayu, Xu Liang. (2017). Discussion on the process of thermal keying for the rotor rim of a large hydroelectric generator [J]. Water Power, 043 (001): 90-93.

[5] Yan Zhi. (2015). Research on the installation technology of the Wedge of the rotor of the hydro-generator unit $[\mathrm{J}]$.
Architectural Engineering Technology and Design, 000 (029): $1160,1179$.

[6] Li Chengfeng. (2013). Assembling technology of generator rotor on the left bank of Xiluodu [J]. Applied Energy Technology, 000 (006): 43-46.

[7] Gong Benchao. (2014). The application of rotor assembly technology in the installation of hydropower generating units $[\mathrm{J}]$. Scientific and technological innovation, 75-75.

[8] Wang Xiuran, Shi Daiyong. (2014). On-site assembly and welding technology of large generator rotor wedge $[\mathrm{J}]$. Mechanical \&Electrical Technology of Hydropower Station, 000 (006): 53-54.

[9] Zhou Ruoyu, Fan Xiaokui, Sun Hongbing. (2015). Measuring device for the gap between the rotor rim and the lwedge of hydropower station, CN204240923U [P].

[10] Chen Yuechun, Yao Gang.(2012). Research on the installation technology of the wedge of the rotor of the hydroelectric generator [J]. Guizhou Electric Power Technology, 45-47.

[11] LvYuzhong, Li Chuanfa, Li Shunfeng. (2011). The characteristics and assembly of the generator rotor of Pubugou Hydropower Station [J]. Advances in science and technology of water resources, 31 (S2): 39-41.

[12] WenYoufu.(2010). Rotor assembly technology of high-head hydro-generator set in Henan Baoquan [J], Yangtze River, 41 (012): 77-79.

[13] Chen Xifang. (2008). Monitoring and maintenance of hydraulic turbine generator structure operation [M]. Beijing: China Water\&Power Press.

[14] Yu Bin. (2017). Discussion on Installation Technology of Wedge of Hydrogenerator Unit [J]. Science \& Technology Vision, 000 (015): 37-38.

[15] Mu Guanhua, Si Yonggang, (2006). Key control measures for rotor assembly of ALSTOM units in Three Gorges Left Bank Power Station [C]. The First International Conference on Hydropower Technology. 